\title{
DEVELOPMENT OF THEORY FOR KERNEL-SHELL EXTRACTION ON ROTATING INCLINE
}

UDC: 621.927 .4

Original scientific paper

https://doi.org/10.18485/aeletters.2020.5.1.5

\author{
Eyitope I. Alade ${ }^{1 *}$, Olufemi A. Koya ${ }^{1}$, Olakunle Kayode ${ }^{2}$, Rasheed A. Adetona ${ }^{3}$ \\ ${ }^{1}$ Department of Mechanical Engineering, Obafemi Awolowo University, Ile-Ife, Nigeria \\ ${ }^{2}$ Department of Mechanical Engineering, Osun State University, Osogbo, Nigeria \\ ${ }^{3}$ Department of Mathematics, Obafemi Awolowo University, lle-Ife, Nigeria
}

\begin{abstract}
:
Separation theory describing the motions of palm kernel and shell on rotating incline was analysed. Two series of differential equations, in order to obtain the dispatch angles of kernel and shell for the separation process were developed respectively. The differences in the motions and physical features of the particles formed the premise for separation. The results obtained from the theory showed a possibility of product separation within the approximate ranges of dispatch angles between 25 and $90^{\circ}$ for kernels and shells on rotating incline, with specified slide limit, angular velocity and radius of the incline, and comparisons were made between the theoretical and experimental results. The dispatch angles obtained from both theory and experiment reasonably showed significant agreement and therefore suggested validity of the developed theory.
\end{abstract}

ARTICLE HISTORY

\section{INTRODUCTION}

Interest in effective palm kernel and shell extractions is growing due to their economic and technical values world over. Works had been done on machine to crack palm nut shells in order to release the kernel undamaged [1-4]. In the recent years, various researchers and engineers had attempted developing different types of prototypes for kernel-shell separation, which are mostly at the experimental stages, and some of them may function mainly as pre-cleaners, to screen out dirts, small shell particles and immature kernels [1,5-12]. Moreover, industrial practices and review of literature indicated that the most suitable technology has not been defined for dry separation system to be applied for commercial purposes.

The product from nut-cracking operation is usually a mixture of kernel and broken shell of varied sizes. The requirements for kernel cleanliness and quality present major challenges in the palm kernel-shell extraction process, especially, to the small-scale mill owners. At present, techniques employed in the separation of kernelshell mixture are of two types: wet and dry methods. Large-scale mills have automated hydrocyclone machines with high separation efficiency; while, clay-baths and hydro cyclones are known for their high energy and water consumption, making their application in small-scale mills unattractive $[6,13,14]$. Moreover, kernels recovered in the wet systems need further preservation against the growth of fungi and re-dried for the removal of absorbed moisture during the separation process. This therefore implies that dry separation system will result in saving the energy required in redrying the kernels separated via the liquid medium [9].

The procedures for dry separation consist of winnowing followed by handpicking, reciprocating inclined plane, vibrating and reciprocating screens, rotating screens, indented cylinders, and pneumatic conveying in a vertical tunnel. The 
conventional hand picking method of kernel-shell separation presents a high efficiency but it is time consuming, tedious, and of very low capacity of about $60 \mathrm{kgh}^{-1}$ [7]. Also, the reported efficiencies for other mechanical systems showed that substantial improvements are indispensable $[6,7,15,16]$. Hence, dry separation of palm kernelshell mixture has remained a tough challenge, particularly in handling kernels and shells of comparable size grades. Where the kernel and shell are of the same size grade, with equal chances of passing through the same aperture, the only distinguishing features are the shapes of the particles and their friction coefficients on the structural surface. It is, also, widely known, based on observations, that the shells are mostly flat or dish-shaped, and that the shape of the kernels vary from being nearly spherical to being ellipsoidal $[7,17]$. Consequently, the difference in the motion of particles of different shapes was considered as a basis for the feasible separation of the particles. This same postulation was considered in the analysis of kernel-shell separation on a spinning disc [9], but the hardware for the realization of the theory may require series of discs, and the material handling capacity may be quite low. It was therefore considered that the use of a rotating incline device could present better realistic solution, in terms of difference in the motions of kernels and shells on the device, taking into consideration the variation in the physical characteristics of the particles, such as shape and frictional coefficient. The focus therefore, of this study was to develop viable concept that may form the basis of evolving the device for effective separation of kernel and shell on dry basis.

\section{SEPARATION THEORY FOR PALM KERNEL AND SHELL}

Based on the differences in the shapes and motions of the particles (palm kernel and shell) along the surface of rotating inclined device, the following assumptions were made:

$>$ kernel will roll off the inclined surface,

$>$ kernel will slide off the surface,

shell will slide down the rotating inclined surface.

\subsection{Rolling particles}

Fig. 1 typifies the motion of particles that purely roll without slipping on the rotating incline. From the figure, for a particle in free rolling:

$$
m g \sin \theta-F_{I}-m \omega^{2} r=m \ddot{x}
$$

Where:

$$
x=n \alpha
$$

$x$ - is displacement; $\alpha$ - is angular displacement; and

Now,

$$
F_{I}=\frac{I \ddot{x}}{n}
$$

Where:

$F_{I^{-}}$is Frictional force; $I$ - is moment of inertial $=m k^{2}$ (where $k$-is radius of gyration); and $n-$ is radius of rolling particles, hence;

$$
m g \sin \theta-I \ddot{\alpha}-m \omega^{2} r=m \ddot{x}
$$

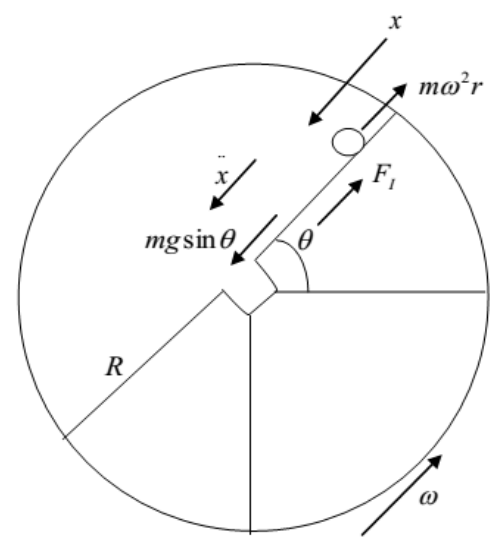

Fig.1. Representation of rolling particle on the rotating incline

Substituting values:

$$
g \sin \omega t-\frac{k^{2} \ddot{x}}{n}-\omega^{2} r=\ddot{x}
$$

Note: $r=R-x$

Where $r$ is radial distance of particle from the centre of incline, and $R$ is radius of the drum

$$
\ddot{x}\left(1+\frac{k^{2}}{n}\right)-\omega^{2} x=g \sin \omega t-\omega^{2} R
$$

Dividing through by $1+\frac{k^{2}}{n}$

Hence;

$$
\ddot{x}-\left(\frac{\omega^{2} n}{n+k^{2}}\right) x=\frac{n g}{n+k^{2}} \sin \omega \mathrm{t}-\frac{n \omega^{2} R}{n+k^{2}}
$$


Let:

$\frac{\omega^{2} n}{n+k^{2}}=\alpha_{1}, \frac{n g}{n+k^{2}}=\beta \quad$ and $\quad \frac{n \omega^{2} R}{n+k^{2}}=\varepsilon$

and assuming a rolling, spherical solid particle, $I=2 m n^{2} / 5$ [18],

$$
k^{2}=\frac{2}{5} n^{2}
$$

Hence,

$$
\begin{aligned}
\frac{5 \omega^{2}}{5+2 n} & =\alpha_{1} \\
\frac{5 g}{5+2 n} & =\beta \\
\frac{5 \omega^{2} R}{5+2 n} & =\varepsilon \\
\ddot{x}-\alpha_{1} x & =\beta \sin \omega t-\varepsilon
\end{aligned}
$$

Let:

$$
\begin{aligned}
x=\lambda^{m t}, \dot{x}= & m \lambda^{m t}, \ddot{x}=m^{2} \lambda^{m t} \\
& m^{2} \lambda^{m t}-\alpha_{1} \lambda^{m t}=0
\end{aligned}
$$

and

$x_{c}=A_{1} \lambda^{\sqrt{\alpha_{1} t}}+A_{2} \lambda^{-\sqrt{\alpha_{1} t}} \Rightarrow A_{1} \cosh \sqrt{\alpha_{1} t}+A_{2} \sinh \sqrt{\alpha_{1} t}$

Now, to determine the particular integral using operation $\mathrm{D}$ method;

$$
x_{p}=\frac{1}{D^{2}-\alpha_{1}}(\beta \sin \omega t-\varepsilon)
$$

The complete solution therefore becomes:

$$
x=x_{c}+x_{p}
$$

$$
x=A_{1} \cosh \sqrt{\alpha_{1} t}+A_{2} \sinh \sqrt{\alpha_{1} t}-\frac{\beta \sin \omega t}{\omega^{2}+\alpha_{1}}+\frac{\varepsilon}{\alpha_{1}}
$$

Applying the boundary conditions; at $t=0$, $x=0$;

Also;

$$
A_{1}=-\frac{\varepsilon}{\alpha_{1}}
$$

$$
\dot{x}=A_{1} \sqrt{\alpha_{1}} \sinh \sqrt{\alpha_{1} t}+A_{2} \sqrt{\alpha_{1}} \cosh \sqrt{\alpha_{1} t}-\frac{\beta \omega \cos \omega t}{\omega^{2}+\alpha_{1}}
$$

At $t=0, \dot{x}=0$, then;

$$
A_{2}=\frac{\beta \omega}{\sqrt{\alpha_{1}}\left(\omega^{2}+\alpha_{1}\right)}
$$

Substituting values for $A_{1}$ and $A_{2}$

$$
\begin{aligned}
x=-\frac{\varepsilon}{\alpha_{1}} \cosh \sqrt{\alpha_{1} t}+ & \frac{\beta \omega}{\sqrt{\alpha_{1}}\left(\omega^{2}+\alpha_{1}\right)} \sinh \sqrt{\alpha_{1} t}- \\
& -\frac{\beta \sin \omega t}{\omega^{2}+\alpha_{1}}+\frac{\varepsilon}{\alpha_{1}}
\end{aligned}
$$

From Equations 8 and 10;

$$
\frac{\varepsilon}{\alpha_{1}}=R
$$

Hence,

$$
\begin{gathered}
x=-R\left[\cosh \sqrt{\alpha_{1} t}-1\right]+ \\
+\frac{\beta}{\left(\omega^{2}+\alpha_{1}\right)}\left[\frac{\omega}{\sqrt{\alpha_{1}}} \sinh \sqrt{\alpha_{1} t}-\sin \omega t\right]
\end{gathered}
$$

Substituting values for $\alpha_{1}$ and $\beta$;

$$
\begin{gathered}
x=\frac{5 g}{2 \omega^{2}(5+n)}\left[\frac{\sinh \sqrt{\frac{5}{5+2 n} \omega t}}{\sqrt{\frac{5}{5+2 n}}}-\sin \omega t\right]- \\
-R\left[\cosh \left(\sqrt{\frac{5}{5+2 n} \omega t}\right)-1\right]
\end{gathered}
$$

\subsection{Sliding particles}

Similarly, considering the sliding particles down the rotating inclined as depicted in Fig.2, the following relationships apply:

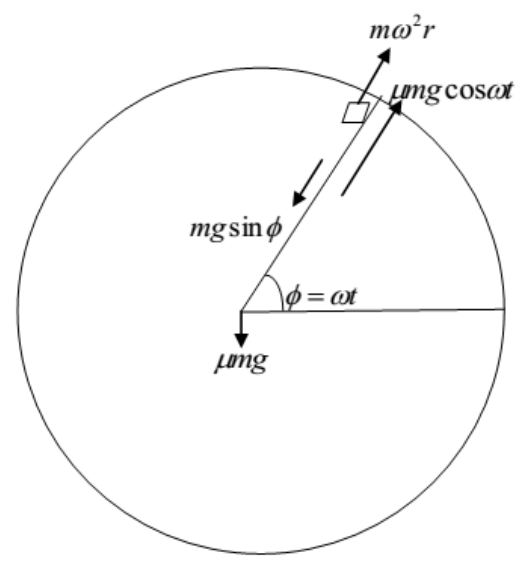

Fig.2. Representation of sliding particle on the rotating incline

$$
m g \sin \omega t-m \omega^{2} r-\mu m g \cos \omega t=m \ddot{x}
$$

Note: $r=R-x$

$$
g \sin \omega t-\mu g \cos \omega t-\omega^{2} R+\omega^{2} x=\ddot{x}
$$

That is,

$$
g(\sin \omega t-\mu \cos \omega t)-\omega^{2} R=\ddot{x}-\omega^{2} x
$$

$$
m^{2} l^{m t}-\omega^{2} l^{m t}=0
$$


With $l^{m t} \neq 0, m^{2}=\omega^{2}, m= \pm \sqrt{\omega^{2}}= \pm \omega$

$$
\begin{gathered}
x_{c}=A l^{\omega t}+B l^{-\omega t} \\
x_{c}=A \cosh (\omega t)+B \sinh (\omega t)
\end{gathered}
$$

Determining particular solution using operation D method;

$$
\begin{gathered}
x_{p=} \frac{1}{D^{2}-\omega^{2}}\left(g \sin \omega t-\mu g \cos \omega t-\omega^{2} R\right) \\
x=A \cosh (\omega t)+B \sinh (\omega t)-\frac{g}{2 \omega^{2}} \sinh (\omega t)+ \\
+\frac{\mu g \cos \omega t}{2 \omega^{2}}+R
\end{gathered}
$$

Applying the boundary conditions;

At $t=0, x=0$

$$
A=-\left[\frac{\mu g}{2 \omega^{2}}+R\right]
$$

Similarly:

$$
\begin{aligned}
& \dot{x}=A \omega \sinh \omega t+B \omega \cosh \omega t-\frac{g \cos \omega t}{2 \omega}-\frac{\mu g \sin \omega t}{2 \omega} \\
& \text { At } t=0, \dot{x}=0 \\
& B=\frac{g}{2 \omega^{2}}
\end{aligned}
$$

The complete solution therefore becomes:

$$
\begin{gathered}
x=x_{c}+x_{p} \\
x=\frac{g}{2 \omega^{2}}[\sinh (\omega t)+\mu \cos \omega t-\sin \omega t]- \\
-\left[\frac{\mu g}{2 \omega^{2}}+R\right] \cosh (\omega t)+R
\end{gathered}
$$

\subsection{Basic significance of analysed theory to kernel and shell separation}

Deducing from the separation theory, the required factors in determining the motions of the particles on rotating incline device are motion and shape of the particle, slide limit, speed of the rotating incline, diameter of the drum, gravitational acceleration and coefficient of friction. Typical values of shapes and coefficient of friction for kernel and shell on some structural surfaces are available in relevant literatures $[9,19]$. Hence, the theoretical dispatch angles can be plotted for the particles in a rotating drum of specified diameter and slide limit, at a constant angular velocity, to obtain the values providing best promise for the separation of the mixture.
The relative motion of particles on a rotating incline are therefore expressed in Equations (23) and (35), assuming the mixture of kernel and shell as aggregate of detached particles with no interaction between them as well as each particle was to be in contact with the incline slide. It was assumed that the motion of each constituent is represented by the motion of a proprietary single particle, and complete separation becomes viable when there are differences in the dispatch angles for the kernels and the shells within the rotating inclined.

\section{MATERIALS AND METHOD}

This section presents the materials as well as methodology employed in the validation of the theoretical analysis presented in the previous section.

\subsection{Typical features of kernel and shell}

Dura variety of palm nut sample, usually classified as thick-shelled with large kernel, resistant to cracking and prone to kernel breakage [15], was selected for this study. It was reported by [16] that typical African dura nut is about $8-20 \mathrm{~mm}$ in diameter and has a fairly uniform shell thickness of about $2 \mathrm{~mm}$. The values of the nut properties required for this study such as coefficients of friction of kernel and shell particles; and estimated radius of rolling particles ' $n$ ' determined from their geometric mean diameter were adopted from [19] and [20] respectively. Other relevant parameters such as radius of the rotating drum, slide size and angular velocity of the rotating incline device were deduced from [21].

The coefficients of friction of 0.68 and 0.95 respectively for kernel and shell were taken. This was premised on the highest values of friction coefficients providing largest difference between kernel and shell from reported experimental observation [9].

\subsection{Device for experimentation}

In order to verify the analysed theory, it was indispensable to fabricate an experimental equipment. The device (Fig.3 and 4) is composed of rotary drum, slides, collector, shaft and conveyor. The development of the device was principally guided by the fundamental separation theory. The drum is made up of expanded metal and fabricated into a regular cylindrical shape of 
$0.508 \mathrm{~m}$ diameter. Two slides of $0.127 \mathrm{~m}$ width are mounted on the drum. The conveyor and rotating shaft welded together are installed in the shell collector of $0.0762 \mathrm{~m}$ (3 inches) diameter segmented pipe as shown.

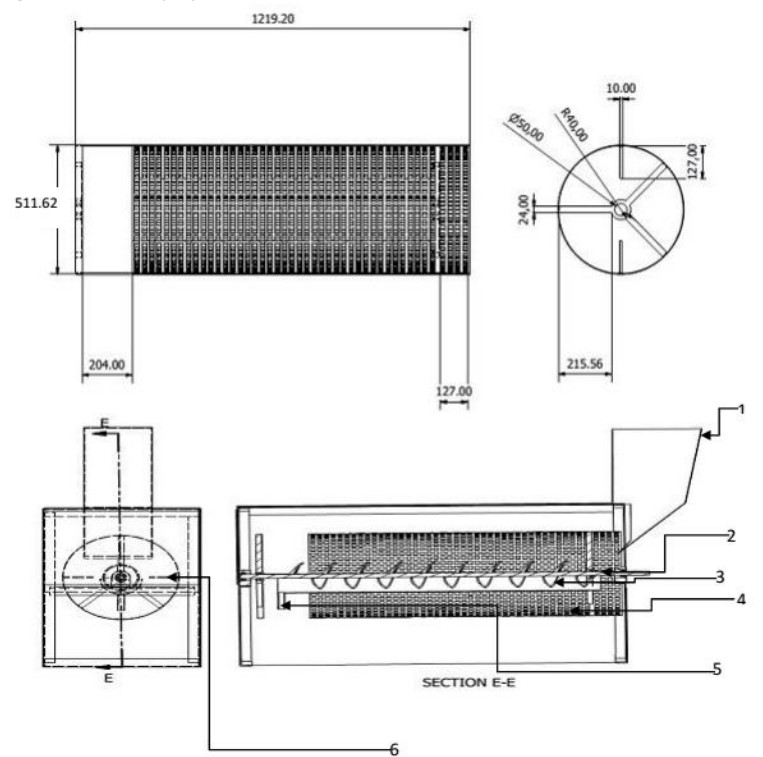

Fig.3. Cross section of the verifying device (1-hopper; 2 shaft; 3-conveyor; 4-rotary drum; 5-collector; 6-slide)

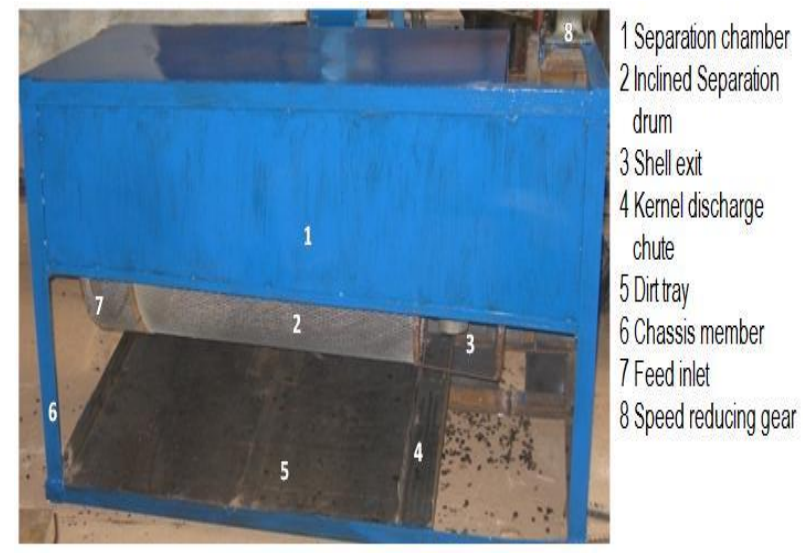

Fig.4. Experimental rotating inclined separator for verification of theory

For a centrifugal motion of the rotating separator, the following relationship among the angular velocity $\omega$, gravitational acceleration $g$, and radius of the drum $r$ applies:

$$
\omega<\sqrt{\frac{g}{r}}
$$

Where; $g=9.81 \mathrm{~m} / \mathrm{s}^{2}$; and designing a prototype drum with diameter $0.508 \mathrm{~m} ; r=0.254 \mathrm{~m}$.

Substituting values hence;

$$
\omega<6.21 \frac{\mathrm{rad}}{\mathrm{s}}
$$

\subsection{Experimental methodology}

Sample of dura variety of palm nut was drawn from large tonnage, which had been sun-dried for commercial kernel and shell extraction. The samples were initially cracked and consequently fed into the developed device in batches, driven below the designed speed limit (Equation 36) of $6.21 \frac{\mathrm{rad}}{\mathrm{s}}$. The dispatch angles were determined by observing the angles at which the particles completely either roll or slide off the rotating slide. This was achieved by periodic operation of the device (dynamically and statically) and measuring the inclined dispatch angles of each trial on the slide to the horizontal plane.

The dispatch angles for the theory were also obtained by substituting the appropriate values into Equations (23) and (35). These were numerically analysed taking diameter of drum as $0.508 \mathrm{~m}$; width of slide as 0.127 [21] and then plotted as shown on Table 1 and Fig. 5 respectively.

\section{RESULTS AND DISCUSSION}

This section presents both the experimental and theoretical results obtained as well as their interpretation

Table 1. Kernel-Shell Displacement on Rotating Inclined Device

\begin{tabular}{|c|c|c|c|}
\hline $\begin{array}{c}\text { Dispatch } \\
\text { Angle }(\text { deg })\end{array}$ & $\begin{array}{c}\text { Kernel } \\
\text { Rolls } \\
(m)\end{array}$ & $\begin{array}{c}\text { Kernel } \\
\text { Slides } \\
(m)\end{array}$ & $\begin{array}{c}\text { Shell } \\
\text { Slides }(m)\end{array}$ \\
\hline 0 & 0 & 0 & 0 \\
\hline 5 & 0.027399 & 0.026595 & 0.026254 \\
\hline 10 & 0.053081 & 0.049743 & 0.048377 \\
\hline 15 & 0.077245 & 0.069625 & 0.066542 \\
\hline 20 & 0.100077 & 0.086395 & 0.08089 \\
\hline 25 & 0.121756 & 0.100185 & 0.091535 \\
\hline 30 & 0.14245 & 0.111106 & 0.098562 \\
\hline 35 & 0.162319 & 0.119243 & 0.102028 \\
\hline 40 & 0.181518 & 0.124662 & 0.101965 \\
\hline 45 & 0.200198 & 0.12741 & 0.098374 \\
\hline 50 & 0.218503 & 0.12751 & 0.091233 \\
\hline 55 & 0.236577 & 0.124968 & 0.080492 \\
\hline 60 & 0.254561 & 0.119767 & 0.066071 \\
\hline 65 & 0.272594 & 0.111873 & 0.047866 \\
\hline 70 & 0.290819 & 0.101228 & 0.025741 \\
\hline 75 & 0.309377 & 0.087756 & -0.00047 \\
\hline 80 & 0.328413 & 0.071358 & -0.03096 \\
\hline 85 & 0.348075 & 0.051912 & -0.06596 \\
\hline 90 & 0.368517 & 0.029274 & -0.10573 \\
\hline
\end{tabular}




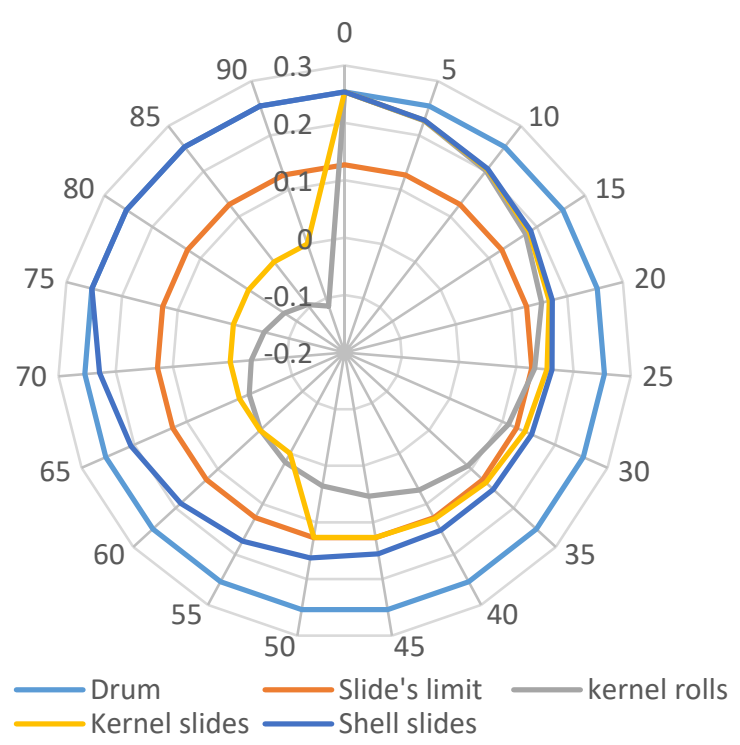

Fig.5. Motions of the particles on the rotating inclined surface

\subsection{Theoretical results}

Table 2 shows the average values of theoretical and experimental dispatch angles of rotating incline concept for kernel and shell separation. The paths of the particles on the inclined showed a complete separation of the products. It is indicated that the rolling and sliding kernels are dispatched between $25-30^{\circ}$ and $40-45^{\circ}$ angles of rotation respectively; while shell particles do not dispatch between $0-90^{\circ}$. This implies shell particles will dispatch by gravity from angle greater or equal to $90^{\circ}$.

\subsection{Experimental results}

Experimental observations showed that the rolling and sliding kernels were dispatched between $20-35^{\circ}$ and $40-50^{\circ}$ angles of rotation respectively; while shell particles were dispatched between $70-90^{\circ}$. However, during continuous operation of the experimental device certain kernels were lost with shells. This was probably due to imprecision in the fabrication of the rotating incline as designed; and it accounted for the loss of some kernels with shells. Also, $100 \%$ of shell particles were recovered in the appropriate compartment as expected. Hence, the possibility of separating kernel from shell using the tested concept was confirmed.

The values showed that the deviation of experimental measurement from theory ranged between 0 and $12.5 \%$, which are reasonable. This was substantiated by [22] that in the applications of machine design, estimation of theoretical deviations ranging from 10 to $45 \%$ are still considered good.

Table 2.Comparison of average dispatch angles of the theory and the experiment

\begin{tabular}{|l|c|c|c|}
\hline \multirow{2}{*}{ Product } & \multicolumn{3}{|c|}{ Average dispatch angles, deg } \\
\cline { 2 - 4 } & Theory & Experiment & Deviation \\
\hline Kernel roll & 27.5 & 27.5 & $0(0 \%)$ \\
\hline $\begin{array}{l}\text { Kernel } \\
\text { slide }\end{array}$ & 42.5 & 45 & $2.5(5.6 \%)$ \\
\hline Shell slide & 90 & 80 & $10(12.5 \%)$ \\
\hline \multicolumn{4}{r|}{ Not significant at $p<0.1 ;$ t-value is 0.580}
\end{tabular}

\section{CONCLUSIONS}

The application of rotating incline device for possible separation of kernel and shell particles was theoretically analysed and a proprietary device was fabricated mainly for experimental verification of the theory. The differences in the motions and physical properties of the particles provided a principle for the product separation. The comparisons between the theoretical and experimental results reasonably showed consistency, therefore supporting the theory and possibility of rotating incline device application in the separation of kernel and shell mixture.

\section{REFERENCES}

[1] J. O. Olaoye, T. Adekanye, Properties influencing cracking and separation of palm nuts in a mechanical cracker cum separator. Croat. J. Food Sci. Technol., 10(1), 2018: 42-50. https://doi.org/10.17508/CJFST.2018.10. 1.07

[2] K.C. Umani, O.M. Olatunji, I.E. Ekop, G.E. Akpan, Experimental investigation of the effect of rotor speed of a horizontal shaft centrifugal palm nut cracker on optimum whole kernel production and shell particle size. Scientific African 7, 2020: e00238.

https://doi.org/10.1016/j.sciaf.2019.e00238

[3] S.B. Udo, A.F. Adisa, S.O. Ismaila, S.B. Adejuyigbe, Performance evaluation of a palm kernel nut cracking machine, J. Nat. Sci. Eng. Technol., 14(1), 2015: 111-116.

[4] F.A. Oluwole, M.B. Oumarou, G.M. Ngala, Dynamics of centrifugal impact nut cracker, Int. J. Res. Stud. Sci. Eng. Technol., 3(1), 2016, 15-21. 
[5] O. Adewale, O. Koya, A rotary separator for the dry mixture of palm kernel and shell. Innov. Systems Design Engin.,5 (7), 2014: 3243.

[6] J.J. Olie, T.D. Tjeng, Kernel recovery in the extraction of palm oil. The Netherlands Stork, Amsterdam, Amstelveen, 1974, pp.3-762.

[7] C.O. Akubuo, B.E. Eje, Palm kernel and shell separator. Biosystems Engineering, 81(2), 2002: 193-199.

https://doi.org/10.1006/bioe.2001.0029

[8] FAO, Small scale palm oil processing in Africa. Food and Agricultural Organisation. Agricultural Services Bulletin, 148, 2005, 1824.

[9] O.A. Koya, M.O. Faborode, Separation theory for palm kernel and shell mixture on a spinning disc. Biosystems Engineering, 95(3), 2006: 405-412.

https://doi.org/10.1016/j.biosystemseng.2006.07. $\underline{005}$

[10] M.H. Rohaya, A.B. Nasrin, Y.M. Chao, A.N. Ma, N. Ravi, A Commercial Scale Implementation of Rolek ${ }^{\text {TM }}$ Palm Nut Cracker Techno-Economic Viability Study for Production of Shell-Free Kernel. Journal of Oil Palm Research, Vol. Special Issue, 2006: 153-167.

[11] J.Y. Amoah, M. Aggey, S. Annumu, Crackedmixture sieving rates and efficiencies in smallscale palm nut processing in Ghana. Ghana Journal of Science, 47, 2007: 69-81.

http://dx.doi.org/10.4314/gjs.v47i1.15926

[12] A. A. Aderinlewo, A. A. Agboola, O. J. Adeosun, E. S. A. Ajisegiri, A. F. Adisa. Investigation of Separation of Palm Kernel and Shell on an Inclined Plane Separator. Acta Technica Corviniensis - Bulletin of Engineering, $\mathrm{XI}(2)$, 2018:135-137.

[13] K. Poku, Small-Scale Palm Oil Processing in Africa, FAO Agricultural Services Bulletin.
Publishing Management Service - Information Division, FAO, Rome, 2002, pp.56.

[14] A.T. Olasumboye, O.A. Koya, A rotary separator for the dry mixture of palm kernel and shell. Innovative Systems Design and Engineering, 5(7), 2014: 32-41.

[15] C.W.S. Hartley, The Oil Palm. Longman Publishers, London, 1977, 432-443.

[16] G.A. Badmus, Design of vertical shaft centrifugal palm nut cracker. Presented to the Nigerian Society of Agriculture. University of Agriculture, Makurdi, Nigeria, 1990, pp.24-48.

[17] O.A. Koya, M.O. Faborode, Mathematical modelling of palm nut cracking based on hertz's theory. Biosystems Engineering, 91(4), 2005: 471-478.

http://dx.doi.org/10.1016/j.biosystemseng.2005.0 $\underline{5.011}$

[18] R. F. Steidel, Jr. Mechanics of Solids, in: Marks' Standard Handbook for Mechanical Engineers, Avallone, E.A. and Baumeister III. T. (Eds.), $10^{\text {th }}$ Edition. McGraw-Hill, New York, 1996, pp. 3-9

[19] O.A. Koya, A. Idowu, M.O. Faborode, Physical properties of palm kernel and shell relevant in nut cracking and product separation. Journal of Agricultural Engineering and Technology, 12, 2004: 27-39.

[20] O.A. Koya, Studies of the mechanics of cracking and centrifugal separation of palm kernel and shell mixture. Unpublished Ph.D Thesis. Obafemi Awolowo University, Ile - Ife, Nigeria, 2004.

[21] E.I. Alade, Performance Modelling of an Integrated Palm-Nut Cracker and Kernel-Shell Separator. Unpublished Ph.D Thesis. Department of Mechanical Engineering, Obafemi Awolowo University, Ile - Ife, Nigeria, 2017.

[22] B.J. Hamrock, B.O. Jacobson, S.R. Schmid, Fundamentals of Machine Elements. McGrawHill Company, 1999, pp.257-272. 